6. M. S. Knebelman, Collineations and motions in generalized spaces, Amer. J. Math. vol. 51 (1928) pp. 527-564.

7. D. D. Kosambi, Collineations in path-space, J. Indian Math. Soc. N.S. vol. 1 (1934) pp. 69-72.

8. Jack Levine, Classification of collineations in projectively and affinely connected spaces of two dimensions, Ann. of Math. vol. 52 (1950) pp. 465-477.

9. Sophus Lie and Friedrich Engel, Theorie der Transformationsgruppen, vol. 3, Leipzig, Teubner, 1893, pp. 71-73, 379-380.

10. Buchin $\mathrm{Su}$, On the isomorphic transformations of $K$-spreads in a Douglas space. I, Science Record vol. 2 (1947) pp. 11-19.

11. - On the isomorphic transformations of $K$-spreads in a Douglas space. II, Science Record vol. 2 (1948) pp. 139-146.

12. - Descriptive collineations in spaces of $K$-spreads, Trans. Amer. Math. Soc. vol. 61 (1947) pp. 495-507.

13. - A characteristic property of affine collineations in a space of $K$-spreads, Bull. Amer. Math. Soc. vol. 54 (1948) pp. 136-138.

14. Kentaro Yano, Lie derivatives in general space of paths, Proc. Imp. Acad. Tokyo vol. 21 (1945) pp. 363-371.

15. - Groups of transformations in generalized spaces, Tokyo, Akademeia Press, 1949.

North Carolina State College

\title{
ON THE CONTINUITY OF PARAMETRIC LINEAR OPERATIONS ${ }^{1}$
}

\section{B. J. PETTIS}

The proofs of the theorems asserting strong continuity for semigroups of linear operations in Banach spaces usually involve measurability and integrability of Banach-space-valued functions [2, pp. 183-184]. ${ }^{2} \mathrm{~A}$ theorem of this type in which the assumptions and the proof are purely topological is given below.

Let $G$ be both a topological space and an additive group, and let $H$ be a subset of $G$. For each $h$ in $H$ let $D(h)$ be all points $g$ in $H$ satisfying these two conditions: (1) $h-g \in H$, (2) for each open set $N_{g}$ about $g$ there is an open set $N_{h}$ about $h$ such that $h-g+\left(H \cap N_{\theta}\right)$ $\supset H \cap N_{h}$. Letting $X$ be a complex linear normed space, a set $\Gamma=[\gamma]$ of bounded complex linear functionals on $X$ is a total set for $E, E$ a subset of $X$, if $\|x\|=\sup [|\gamma(x)|, \gamma \in \Gamma]$ holds for every $x$ in the smallest linear subspace containing $E$. A function $T_{h}$ on $H$ to the

Presented to the Society, April 21, 1951; received by the editors May 29, 1950.

${ }^{1}$ This paper was written under Contract N7-onr-434, Task Order III, Navy Department (The Office of Naval Research).

${ }^{2}$ Numbers in brackets refer to the references at the end of the paper. 
space $B(X)$ of bounded linear operations on $X$ to $X$ is additive if $T_{h+k}=T_{h}\left(T_{k}\right)$ whenever $h, k$, and $h+k$ are in $H$. Finally, we define a function $\phi(a)$ on a topological space $A$ to a metric space $B$ to be residually separably-valued if the set $\phi\left(A^{\prime}\right) \equiv\left[\phi(a) \mid a \in A^{\prime}\right]$ is separable in $B$ for some set $A^{\prime}$ residual in $A$, that is, for some $A^{\prime}$ having its complement of the first category.

TheOREM. Let $H$ be a subset of $G$ and let $T_{h}$ be additive on $H$ to $B(X)$. Suppose that (i) $D(h)$ is second category for each $h \in H$, and that (ii) for each $x_{0}$ in $X$ the function $\phi(h) \equiv T_{h}\left(x_{0}\right)$ on $H$ to $X$ is residually separably-valued and there is a total set $\Gamma\left(x_{0}\right)$ for $\phi(H)$ such that $\gamma(\phi(h))$ is continuous on $H$ to complex numbers for each $\gamma \in \Gamma\left(x_{0}\right)$. Then $\phi(h)$ is continuous on $H$ to $X$ for each $x_{0} \in X$.

Fixing $x_{0}$ in $X$, the idea of the proof is to show, using (ii), that the set $C$ of points of continuity of $\phi$ is residual, and then to apply (i) to show that $C=H$.

The proof that $C$ is residual is due to Alexiewicz and Orlicz $[1, \mathrm{pp}$. 107-108 and 114-115] in a slightly more restricted case. Their arguments can be adapted here as follows. We first observe that if $S$ is any closed sphere in $X$ with center $y$ in $\phi(H)$ and radius $r$, then $\phi^{-1}(S)$ is closed in $H$; for since $\Gamma\left(x_{0}\right)$ is a total set for $\phi(H)$ and $\gamma(\phi(h))$ is continuous for $\gamma \in \Gamma\left(x_{0}\right)$, clearly $\phi^{-1}(S)=H[h \mid \| \phi(h)$ $-y|| \leqq r]=H\left[h\left|\sup _{\gamma}\right| \gamma(\phi(h))-\gamma(y) \mid \leqq r\right]=\bigcap_{\gamma} H[h|| \gamma(\phi(h))-\gamma(y) \mid$ $\leqq r]$, a closed set. To establish that $C$ is residual it is enough to prove this: if $\phi$ is any function on a topological space $H$ to a metric space $X$ and $\phi$ is residually separably-valued and $\phi^{-1}(S)$ is closed whenever $S$ is a closed sphere with center in $\phi(H)$, then $\phi$ has its points of continuity forming a residual set $C$. Let $R$ be a residual set such that $\phi(R)$ is separable in $X$. Since $\phi(R)$ is separable, there exist countably many closed spheres $\left\{S_{n}\right\}$ with centers in $\phi(R)$ such that for any open set $V$ in $X$ we have $V \cap \phi(R)=\bigcup\left[S_{n} \cap \phi(R) \mid S_{n} \subset V\right]=\mathrm{U}_{k}\left(S_{n_{k}} \cap \phi(R)\right)$ where $n_{k}$ ranges over all $n$ such that $S_{n} \subset V$. Let $R^{\prime}$ be the complement of $R$ in $H$ and set $P_{V}=R^{\prime} \cap \phi^{-1}(V)$. Then $\phi^{-1}(V)=P_{V}$ $\cup\left[R \cap \phi^{-1}(V)\right] \subset P_{V} \cup \phi^{-1}(V \cap \phi(R)) \subset P_{V} \cup\left(\bigcup_{k} \phi^{-1}\left(S_{n_{k}}\right)\right) \subset \phi^{-1}(V)$, since each $S_{n_{k}} \subset V$; hence $\phi^{-1}(V)=P_{V} \cup\left(\bigcup_{k} F_{n_{k}}\right)$, where $F_{n_{k}}=\phi^{-1}\left(S_{n_{k}}\right)$, and in particular $U_{k}\left(F_{n_{k}}^{0}\right) \subset \phi^{-1}(V)^{0}$, where $E^{0}$ denotes the interior of any set $E$. Now set $Q=R^{\prime} \cup\left(\cup_{n}\left(F_{n}-F_{n}^{0}\right)\right)$; since $R$ is residual and each $F_{n}$ is closed, $Q$ is a first category set. Moreover, clearly $\phi^{-1}(V) \subset P_{V} \cup\left(\bigcup_{k}\left(F_{n_{k}}-F_{n_{k}}^{0}\right)\right) \cup\left(\bigcup_{k} F_{n_{k}}^{0}\right) \subset Q \cup \phi^{-1}(V)^{0}$. Now in $H$ consider any point $g$ not in $Q$ and any open $V$ containing $\phi(g)$; since $g \in \phi^{-1}(V) \subset Q \cup \phi^{-1}(V)^{0}$ and $g \notin Q$, obviously $g \in \phi^{-1}(V)^{0}$ and hence $\phi$ is continuous at $g$. Thus $C$ is residual. 
Let $h$ be fixed in $H$. Since $D(h)$ is second category there exists a point $g$ in $D(h)$ at which $\phi$ is continuous. Set $\rho=\left\|T_{h-g}\right\|$ and let $\epsilon>0$ be given. Having $\phi$ continuous at $g$, there is an open set $N_{g}$ about $g$ such that $\|\phi(k)-\phi(g)\|<\epsilon / \rho$ whenever $k \in H \cap N_{g}$. Then, since $g \in D(H)$, there is an open set $N_{h}$ about $h$ such that $h-g+\left(H \cap N_{g}\right) \supset H \cap N_{h}$. Consider any $h^{\prime}$ in $H \cap N_{h}$. Writing $h^{\prime}=h-g+k$ where $k \in H \cap N_{g}$, we have $\left\|\phi\left(h^{\prime}\right)-\phi(h)\right\|=\|\phi(h-g+k)-\phi(h-g+g)\|=\| T_{h-g}\left(T_{k}\left(x_{0}\right)\right)$ $-T_{h-g}\left(T_{g}\left(x_{0}\right)\right)\|\leqq \rho\| \phi(k)-\phi(g) \|<\epsilon$, showing that $\phi$ is continuous at $h$ and ending the proof.

It is easy to verify that assumption (i) in the theorem is satisfied when $\left(\mathrm{i}^{\prime}\right)$ the group sum $h+k$ in $G$ is continuous in $k$ and for each $h$ in $H$ the set $H \cap(-H+h)$ is in the interior of $H$ and is second category. Statement $\left(\mathrm{i}^{\prime}\right)$ in turn is implied by this; $\left(\mathrm{i}^{\prime \prime}\right) h+k$ is continuous in each variable in $G, G$ is second category, $H$ and $-H$ are open, and $H \subset H+H$. Condition ( $\left.i^{\prime \prime}\right)$ holds, for example, when $G$ is a second category linear topological space and $H$ is an open convex set having the zero element as a limit point. Assumption (ii) of the theorem follows if $H$ contains a countable dense subset and $\gamma\left(T_{h}\left(x_{0}\right)\right)$ is continuous on $H$ to complex numbers for each $x_{0}$ in $X$ and each bounded linear functional $\gamma$ on $X$. In particular, Theorem 9.2.2 of [2] now results.

\section{REFERENCES}

1. A. Alexiewicz and W. Orlicz, Sur la continuité et la classification de Baire des fonctions abstraits, Fund. Math. vol. 35 (1948) pp. 105-126.

2. Einar Hille, Functional analysis and semi-groups, Amer. Math. Soc. Colloquium Publications, vol. 31, New York, 1948.

TUlane ÚNIVERSITY AND

Princeton University 